\title{
Synthesis and Evaluation of Antibacterial and Antifungal Activities of 1,3-Disubstituted-4- thioxoimidazolidin-2-one Derivatives
}

\author{
Marwa A. M. Sh. El-Sharief, ${ }^{1,2, *}$ Samir Y. Abbas, ${ }^{3, \# ~ Z i a d ~ M o u s s a, ~}{ }^{4}$ Eman W. El-Gammal, ${ }^{5}$ Ahmed M. Sh. El-Sharief
}

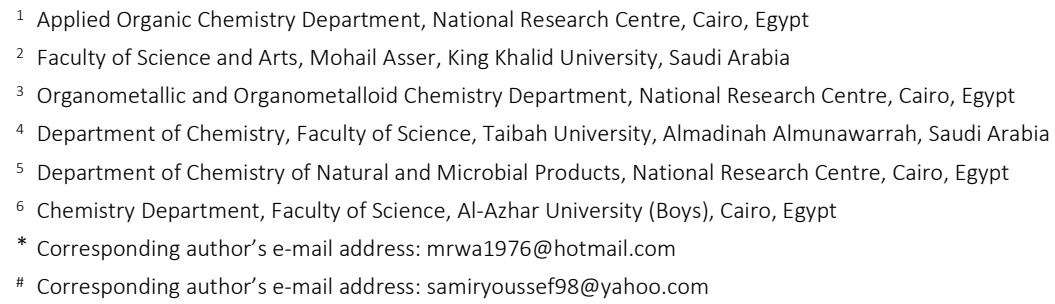

RECEIVED: April 15, 2018 * REVISED: July 2, 2018 * ACCEPTED: July 5, 2018

Abstract: 1,3-Disubstituted-4-thioxoimidazolidin-2-one derivatives with various substituents at $N^{1}$ and $N^{3}$ were synthesized with high yields and excellent purity by the reaction of various $N$-arylcyanothioformamide derivatives with isocyanate derivatives. The activity of these products as antibacterial and antifungal agents was studied to through some light on structural activity relationship. Some of synthesized compounds showed significant antibacterial and antifungal activities. Most of the imidazole derivatives possess significant antifungal activity aginst $S$. cerevisiae (MIC 1-10 $\mu \mathrm{g} \mathrm{mL}^{-1}$ ). As comparision with ketoconazole, most of the imidazole derivatives showed activity ranging from $50 \%$ less activity to fourfold activity.

Keywords: $N$-arylcyanothioformamides, imidazolidineiminothiones, isocyanates, antimicrobial activity.

\section{INTRODUCTION}

MIDAZOLE derivatives are keys compounds in many bioactive compounds of natural and synthetic origins. Nitroimidazoles are a well-known family of antibacterial and antiprozoal drugs, including anti-trypanosomal drugs or compounds with known anti-trypanosomal activity. ${ }^{[1]}$ For more than 50 years, metronidazole was the first drug introduced as antiparasit agent and it was the best-known drug in this class. ${ }^{[2]}$ Imidazolidineiminothione derivatives and their related heterocycles exhibit interesting pharmacological effects such as antibacterial, antifungal antiviral and antitumor agents. ${ }^{[3-7]}$

Bacterial infection and fungal infection are among the widespread and serious diseases. To overcome these serious diseases, the discovery of new types of antimicrobial agents is a very important task. Recently, the research has been focused on the development of new antimicrobial agents, which may act through novel targets and structure design. ${ }^{[8-10]}$

In light of these facts, the present study was designed to synthesize new imidazole derivatives and evaluate their antimicrobial activity. As a trial to obtain novel class of antibacterial and antifungal agents, various groups were introduced into the target compounds.

\section{EXPERIMENTAL}

IR spectra were recorded $(\mathrm{KBr})$ on a Perkin Elmer 1650 spectrophotometer. ${ }^{1} \mathrm{H} N M R$ and ${ }^{13} \mathrm{C}$ NMR spectra were recorded on Avance II Bruker FT NMR spectrometer 400 (400 MHz) using $\mathrm{CDCl}_{3}$ as solvents and TMS as an internal standard. $\mathrm{CFCl}_{3}$ was used as an internal standard for all ${ }^{19} \mathrm{~F}$ NMR measurements. Chemical shifts are expressed as $\delta /$

(c) Br This work is licensed under a Creative Commons Attribution 4.0 International License. 
ppm units. NMR spectra were recorded at Department of Chemistry, Faculty of Science, Taibah University, Almadinah Imunawarrah, Saudi Arabia Mass spectrawere recorded on Shimadzu GC-MS QP 100 EX (70 eV) at the Micro Analytical Center at Cairo University. Melting points were obtained on a Fishere Johns melting points apparatus and are uncorrected.

\section{General Procedure for the Synthesis of Imidazolidineiminothione Derivatives $2 a-h$}

Solution of the cyanothioformanilide derivative $((3,5-$ dichlorophenyl)carbamothioyl cyanide) $(0.01 \mathrm{~mol})$ and the corresponding isocyanate derivatives $(0.01 \mathrm{~mol})$ in dry ether $(30 \mathrm{~mL})$ was treated with three drops of triethylamine. The reaction mixture was stirred for $15 \mathrm{~min}$. The obtained product was filtered off, washed with ether, air-dried and recrystallized from chloroform / $n$-hexane to give imidazolidineiminothione derivatives $\mathbf{2 a}-\mathbf{h}$.

\section{1-(3,5-DICHLOROPHENYL)-5-IMINO-3-PHENYL-4- THIOXOIMIDAZOLIDIN-2-ONE (2a)}

Yield 88 \%; mp 160-162 ${ }^{\circ} \mathrm{C}$; IR: $v / \mathrm{cm}^{-1}$ : $3221(\mathrm{NH}), 1756$ $(\mathrm{C}=\mathrm{O}), 1645$ (C=N), 1126 (C=S); ${ }^{1} \mathrm{H}$ NMR (d1-TFA $/ \mathrm{CDCl}_{3} ; 1 / 1$, $400 \mathrm{MHz}): \delta / \mathrm{ppm}=7.41-7.48(\mathrm{~m}, 2 \mathrm{H}, \mathrm{Ar}-\mathrm{H}), 7.49-7.52$ (m, 2H, Ar-H), 7.62-7.67 (m, 3H, Ar-H), $7.71(\mathrm{t}, 1 \mathrm{H}, J=1.8$ $\mathrm{Hz}, \mathrm{Ar}-\mathrm{H}$ ), 9.53 (br, $1 \mathrm{H}, \mathrm{NH}) ;{ }^{13} \mathrm{C} \mathrm{NMR}$ (d1-TFA / $\mathrm{CDCl}_{3} ; 1 / 1$, $100 \mathrm{MHz}$ ): $125.7(\mathrm{CH}), 126.8(2 \mathrm{CH}), 129.3(\mathrm{C}-\mathrm{N}), 130.6$ $(2 \mathrm{CH}), 131.0(\mathrm{C}-\mathrm{N}), 131.6(2 \mathrm{CH}), 133.2(\mathrm{CH}), 138.1(2 \mathrm{C}-\mathrm{Cl})$, $150.2(\mathrm{C}=\mathrm{N}), 152.0(\mathrm{C}=\mathrm{O}), 176.3(\mathrm{C}=\mathrm{S}) ; \mathrm{MS}(\mathrm{m} / \mathrm{z}: \%): 350\left(\mathrm{M}^{+}\right.$, 20.1), 135 (100); Anal. Calcd for $\mathrm{C}_{15} \mathrm{H}_{9} \mathrm{Cl}_{2} \mathrm{~N}_{3} \mathrm{OS}$ (350.22): C, 51.44; H, 2.59; N, 12.00; Found: C, 51.47; H, 2.61; N, 12.14 .

\section{1-(3,5-DICHLOROPHENYL)-3-(4-FLUOROPHENYL)-5-} IMINO-4-THIOXOIMIDAZOLIDIN-2-ONE (2b)

Yield $84 \%$; mp 136-138 ${ }^{\circ} \mathrm{C}$; IR: $v / \mathrm{cm}^{-1}$ : $3242(\mathrm{NH}), 1765$ $(\mathrm{C}=\mathrm{O}), 1644(\mathrm{C}=\mathrm{N}), 1109$ (C=S); ${ }^{1} \mathrm{H}$ NMR (d1-TFA /CDCl $;$ 1/1, $400 \mathrm{MHz}): \delta / \mathrm{ppm}=7.25-7.36(\mathrm{~m}, 2 \mathrm{H}, \mathrm{Ar}-\mathrm{H}) 7.43-7.48(\mathrm{~m}$, $2 \mathrm{H}, \mathrm{Ar}-\mathrm{H}), 7.49$ (d, $2 \mathrm{H}, J=1.8 \mathrm{~Hz}, \mathrm{Ar}-\mathrm{H}), 7.71(\mathrm{t}, 1 \mathrm{H}, J=1.8$ $\mathrm{Hz}, \mathrm{Ar}-\mathrm{H}$ ), $9.51(\mathrm{br}, 1 \mathrm{H}, \mathrm{NH}) ;{ }^{13} \mathrm{C}$ NMR (d1-TFA $/ \mathrm{CDCl}_{3} ; 1 / 1$, $100 \mathrm{MHz}$ ): 117.7 (d, $J=23.5 \mathrm{~Hz}, \mathrm{CH}$ ); $125.7(\mathrm{CH}), 126.8$ (d, $J=$ $3.2 \mathrm{~Hz}, \mathrm{C}-\mathrm{N}), 129.1$ (d, J = 9.6 Hz, CH), $129.2(\mathrm{C}-\mathrm{N}), 133.2$ (2CH), $138.0(2 \mathrm{C}-\mathrm{Cl}), 150.3(\mathrm{C}=\mathrm{N}), 152.0(\mathrm{C}=\mathrm{O}), 164.3(\mathrm{~d}, \mathrm{~J}=$ 253.4 Hz, C-F), 176.4 (C=S); ${ }^{19} \mathrm{~F}$ (DMSO, 376.5 MHz): -108.5; Anal. Calcd for $\mathrm{C}_{15} \mathrm{H}_{8} \mathrm{Cl}_{2} \mathrm{FN}_{3} \mathrm{OS}$ (368.21): C, 48.93; $\mathrm{H}, 2.19 ; \mathrm{N}$, 11.41; Found: $\mathrm{C}, 48.87 ; \mathrm{H}, 2.21 ; \mathrm{N}, 11.38$.

\section{1-(4-CHLOROPHENYL)-3-(3,5-DICHLOROPHENYL)-4- IMINO-5-THIOXOIMIDAZOLIDIN-2-ONE (2c)}

Yield 89 \%; mp 214-215 ${ }^{\circ} \mathrm{C}$; IR: $v / \mathrm{cm}^{-1}: 3234(\mathrm{NH}), 1766$ (C=O), 1654 (C=N), 1102 (C=S); ${ }^{1} \mathrm{H}$ NMR (d1-TFA / $\mathrm{CDCl}_{3} ; 1 / 1$, $400 \mathrm{MHz}$ ): $\delta / \mathrm{ppm}=7.40$ (d, 2H, J=8.8 Hz, Ar-H), 7.46 (d, $2 \mathrm{H}, J=1.8 \mathrm{~Hz}, \mathrm{Ar}-\mathrm{H}), 7.62(\mathrm{~d}, 2 \mathrm{H}, J=8.8 \mathrm{~Hz}, \mathrm{Ar}-\mathrm{H}), 7.69(\mathrm{t}$,
$1 \mathrm{H}, J=1.8 \mathrm{~Hz}, \mathrm{Ar}-\mathrm{H}), 9.52(\mathrm{br}, 1 \mathrm{H}, \mathrm{NH}) ;{ }^{13} \mathrm{C}$ NMR (d1-TFA $\left./ \mathrm{CDCl}_{3} ; 1 / 1,100 \mathrm{MHz}\right): 125.5(\mathrm{CH}), 128.0(\mathrm{CH}), 129.0(\mathrm{C}-\mathrm{N})$, $129.1(\mathrm{C}-\mathrm{N}), 130.7(\mathrm{CH}), 133.0(\mathrm{CH}), 137.7(\mathrm{C}-\mathrm{Cl}), 137.8(\mathrm{C}-$ $\mathrm{Cl}), 150.0(\mathrm{C}=\mathrm{N}), 151.4(\mathrm{C}=\mathrm{O}), 175.8(\mathrm{C}=\mathrm{S})$; $\mathrm{MS}(\mathrm{m} / \mathrm{z}$ : \%): $385\left(\mathrm{M}^{+}, 25.9\right), 169$ (100); Anal. Calcd for $\mathrm{C}_{15} \mathrm{H}_{8} \mathrm{Cl}_{3} \mathrm{~N}_{3} \mathrm{OS}$ (384.67): C, 46.84; H, 2.10; N, 10.92; Found: C, 46.94; H, 2.12; N, 10.87 .

\section{1-(4-BROMOPHENYL)-3-(3,5-DICHLOROPHENYL)-4- IMINO-5-THIOXOIMIDAZOLIDIN-2-ONE (2d)}

Yield 84 \%; mp 200-202 ${ }^{\circ} \mathrm{C}$; IR: $v / \mathrm{cm}^{-1}$ : $3240(\mathrm{NH}), 1777$ $(\mathrm{C}=0), 1660$ (C=N), 1126 (C=S); ${ }^{1} \mathrm{H}$ NMR (d1-TFA $/ \mathrm{CDCl}_{3} ; 1 / 1$, $400 \mathrm{MHz}$ ): $\delta / \mathrm{ppm}=7.35$ (d, 2H, J = 8.8 Hz, Ar-H), 7.49 (d, $2 \mathrm{H}, J=1.7 \mathrm{~Hz}, \mathrm{Ar}-\mathrm{H}), 7.71(\mathrm{t}, 1 \mathrm{H}, J=1.7 \mathrm{~Hz}, \mathrm{Ar}-\mathrm{H}), 7.79(\mathrm{~d}$, $2 \mathrm{H}, J=8.8 \mathrm{~Hz}, \mathrm{Ar}-\mathrm{H}), 9.49(\mathrm{br}, 1 \mathrm{H}, \mathrm{NH}) ;{ }^{13} \mathrm{C} \mathrm{NMR}$ (d1-TFA $\left./ \mathrm{CDCl}_{3} ; 1 / 1,100 \mathrm{MHz}\right): 125.7(\mathrm{CH}), 126.0(\mathrm{C}-\mathrm{Br}), 128.3(\mathrm{CH})$, $129.2(\mathrm{C}-\mathrm{N}), 129.8(\mathrm{C}-\mathrm{N}), 133.2(\mathrm{CH}), 133.9(\mathrm{CH}), 138.0(\mathrm{C}-$ $\mathrm{Cl}), 150.3(\mathrm{C}=\mathrm{N}), 151.7(\mathrm{C}=\mathrm{O}), 176.0(\mathrm{C}=\mathrm{S})$; $\mathrm{MS}(\mathrm{m} / \mathrm{z}$ : \%): $429\left(\mathrm{M}^{+}, 19.1\right), 215$ (100); Anal. Calcd for $\mathrm{C}_{15} \mathrm{H}_{8} \mathrm{BrCl}_{2} \mathrm{~N}_{3} \mathrm{OS}$ (429.12): C, 41.98; H, 1.88; N, 9.79; Found: C, 42.11; H, 1.82; N, 9.84 .

\section{1-(3,5-DICHLOROPHENYL)-5-IMINO-3-(4-IODOPHENYL)-4- THIOXOIMIDAZOLIDIN-2-ONE (2e)}

Yield 86 \%; mp 189-190 ${ }^{\circ} \mathrm{C}$; IR: $v / \mathrm{cm}^{-1}$ : $3227(\mathrm{NH}), 1771$ $(\mathrm{C}=\mathrm{O}), 1659$ (C=N), 1120 (C=S); ${ }^{1} \mathrm{H}$ NMR (d1-TFA /CDCl 3 ; 1/1, $400 \mathrm{MHz}$ ): $\delta / \mathrm{ppm}=7.20$ (d, $2 \mathrm{H}, J=8.0 \mathrm{~Hz}, \mathrm{Ar}-\mathrm{H}), 7.43$ $7.52(\mathrm{~m}, 3 \mathrm{H}, \mathrm{Ar}-\mathrm{H}), 8.02(\mathrm{~d}, 2 \mathrm{H}, J=8.0 \mathrm{~Hz}, \mathrm{Ar}-\mathrm{H}), 9.50(\mathrm{br}$, $1 \mathrm{H}, \mathrm{NH}) ;{ }^{13} \mathrm{C} \mathrm{NMR}$ (d1-TFA /CDCl $; 1 / 1,100 \mathrm{MHz}$ ): $97.4 \mathrm{C}-\mathrm{I}$ ), $125.8(\mathrm{CH}), 128.3(\mathrm{CH}), 129.2 \mathrm{C}-\mathrm{N}), 130.5 \mathrm{C}-\mathrm{N}), 133.3(\mathrm{CH})$, $138.0 \mathrm{C}-\mathrm{Cl}), 140.0(\mathrm{CH}), 150.3(\mathrm{C}=\mathrm{N}), 151.7(\mathrm{C}=\mathrm{O}), 176.0$ (C=S); MS ( $m$ / z: \%): 474 (M+, 39.4), 260 (100); Anal. Calcd for $\mathrm{C}_{15} \mathrm{H}_{8} \mathrm{Cl}_{2} \mathrm{IN}_{3} \mathrm{OS}$ (476.12): C, 37.84; $\mathrm{H}, 1.69 ; \mathrm{N}, 8.83$; Found: $\mathrm{C}, 37.74 ; \mathrm{H}, 1.71 ; \mathrm{N}, 8.91$.

\section{1-(3,5-DICHLOROPHENYL)-5-IMINO-4-THIOXO-3-P- TOLYLIMIDAZOLIDIN-2-ONE (2f) ${ }^{[6]}$}

Yield 88 \%; mp 207-208 ${ }^{\circ} \mathrm{C}$; IR: $v / \mathrm{cm}^{-1}$ : $3240(\mathrm{NH}), 1771$ (C=O), 1667 (C=N), 1121 (C=S); ${ }^{1} \mathrm{H}$ NMR (d1-TFA /CDCl 3 ; 1/1, $400 \mathrm{MHz}$ ): $\delta / \mathrm{ppm}=2.48\left(\mathrm{~s}, 3 \mathrm{H}, \mathrm{CH}_{3}\right), 7.32(\mathrm{~d}, 2 \mathrm{H}, J=8.3$ $\mathrm{Hz}, \mathrm{Ar}-\mathrm{H}$ ), 7.44 (d, $2 \mathrm{H}, J=8.3 \mathrm{~Hz}, \mathrm{Ar}-\mathrm{H}), 7.50$ (d, $2 \mathrm{H}, J=1.8$ $\mathrm{Hz}, \mathrm{Ar}-\mathrm{H}), 7.70(\mathrm{t}, 1 \mathrm{H}, J=1.8 \mathrm{~Hz}, \mathrm{Ar}-\mathrm{H}), 9.51(\mathrm{br}, 1 \mathrm{H}, \mathrm{NH})$; ${ }^{13} \mathrm{C} \mathrm{NMR}$ (d1-TFA / $\mathrm{CDCl}_{3} ; 1 / 1,100 \mathrm{MHz}$ ): $20.9\left(\mathrm{CH}_{3}\right), 125.7$ (CH), $126.5(\mathrm{CH}), 128.2(\mathrm{C}-\mathrm{N}), 129.3(\mathrm{C}-\mathrm{N}), 131.1(\mathrm{CH})$, $133.2(\mathrm{CH}), 138.0(\mathrm{C}-\mathrm{Me}), 142.6(\mathrm{C}-\mathrm{Cl}), 150.2(\mathrm{C}=\mathrm{N}), 152.4$ $(\mathrm{C}=0), 176.4(\mathrm{C}=\mathrm{S})$; $\mathrm{MS}\left(\mathrm{m} / \mathrm{z}\right.$ : \%): $364\left(\mathrm{M}^{+}, 24.3\right), 91(100)$; Anal. Calcd for $\mathrm{C}_{16} \mathrm{H}_{11} \mathrm{Cl}_{2} \mathrm{~N}_{3} \mathrm{OS}$ (364.25): C, 52.76; H, 3.04; N, 11.54; Found: C, 52.85; H, 3.07; N, 11.62 .

\section{1-(3,5-DICHLOROPHENYL)-3-(2,4-DIMETHOXYPHENYL)-5- IMINO-4-THIOXOIMIDAZOLIDIN-2-ONE (2g)}

Yield 79 \%; mp 154-156 ${ }^{\circ} \mathrm{C}$; IR: $v / \mathrm{cm}^{-1}$ : $3244(\mathrm{NH}), 1772$ $(\mathrm{C}=0), 1645(\mathrm{C}=\mathrm{N}), 1108$ (C=S); ${ }^{1} \mathrm{H}$ NMR (d1-TFA $/ \mathrm{CDCl}_{3} ; 1 / 1$, 
$400 \mathrm{MHz}): \delta / \mathrm{ppm}=3.87\left(\mathrm{~s}, 3 \mathrm{H}, \mathrm{OCH}_{3}\right), 3.98\left(\mathrm{~s}, 3 \mathrm{H}, \mathrm{OCH}_{3}\right)$, 6.73-6.79 (m, 1H, Ar-H), 7.25-7.32 (m, 2H, Ar-H), 7.49 (d, $2 \mathrm{H}, J=1.8 \mathrm{~Hz}, \mathrm{Ar}-\mathrm{H}), 7.69(\mathrm{t}, 1 \mathrm{H}, J=1.8 \mathrm{~Hz}, \mathrm{Ar}-\mathrm{H}), 9.49(\mathrm{br}$, $1 \mathrm{H}, \mathrm{NH}$ ); ${ }^{13} \mathrm{C}$ NMR (d1-TFA / $\mathrm{CDCl}_{3} ; 1 / 1,100 \mathrm{MHz}$ ): 56.1 $\left(2 \mathrm{CH}_{3}\right), 100.6(\mathrm{CH}), 125.7(\mathrm{CH}), 129.3(\mathrm{C}-\mathrm{N}), 129.6(\mathrm{CH})$, $129.7(\mathrm{CH}), 133.1(\mathrm{CH}), 138.0(\mathrm{C}-\mathrm{Cl}), 150.2(\mathrm{C}=\mathrm{N}), 152.0$ $(\mathrm{C}=\mathrm{O}), 155.9$ (C-O), 156.0 (C-O), 176.0 (C=S); MS ( $\mathrm{m} / \mathrm{z}: \%)$ : $410\left(\mathrm{M}^{+}, 31.5\right), 189$ (100); Anal. Calcd for $\mathrm{C}_{17} \mathrm{H}_{13} \mathrm{Cl}_{2} \mathrm{~N}_{3} \mathrm{O}_{3} \mathrm{~S}$ (410.27): C, 49.77; H, 3.19; N, 10.24; Found: C, 49.83; H, $3.21 ; \mathrm{N}, 10.31$.

\section{1-(3,5-DICHLOROPHENYL)-3-(4-ETHOXYPHENYL)-5- IMINO-4-THIOXOIMIDAZOLIDIN-2-ONE (2h)}

Yield $81 \%$; mp 178-180 ${ }^{\circ} \mathrm{C}$; IR: $v / \mathrm{cm}^{-1}$ : $3240(\mathrm{NH}), 1777$ $(\mathrm{C}=\mathrm{O}), 1660(\mathrm{C}=\mathrm{N}), 1126$ (C=S); ${ }^{1} \mathrm{H}$ NMR (d1-TFA $/ \mathrm{CDCl}_{3} ; 1 / 1$, $400 \mathrm{MHz}): \delta / \mathrm{ppm}=1.51\left(\mathrm{t}, 3 \mathrm{H}, J=7.0 \mathrm{~Hz}, \mathrm{CH}_{2} \underline{\mathrm{CH}_{3}}\right), 4.26$ (q, $\left.2 \mathrm{H}, J=7.0 \mathrm{~Hz}, \mathrm{CH}_{2} \mathrm{CH}_{3}\right), 7.19(\mathrm{~d}, 2 \mathrm{H}, J=9.0 \mathrm{~Hz}, \mathrm{Ar}-\mathrm{H}$ ), $7.40(\mathrm{~d}, 2 \mathrm{H}, J=9.0 \mathrm{~Hz}, \mathrm{Ar}-\mathrm{H}), 7.50(\mathrm{~d}, 2 \mathrm{H}, J=1.7 \mathrm{~Hz}, \mathrm{Ar}-\mathrm{H})$, $7.71(\mathrm{t}, 1 \mathrm{H}, J=1.7 \mathrm{~Hz}, \mathrm{Ar}-\mathrm{H}), 9.56(\mathrm{br}, 1 \mathrm{H}, \mathrm{NH}) ;{ }^{13} \mathrm{C} \mathrm{NMR}$ (d1TFA / $\left.\mathrm{CDCl}_{3} ; 1 / 1,100 \mathrm{MHz}\right): 14.1\left(\mathrm{CH}_{3}\right), 65.6\left(\mathrm{CH}_{2}\right), 116.7$ (CH), $124.0(\mathrm{C}-\mathrm{N}), 125.7(\mathrm{C}-\mathrm{H}), 128.4(\mathrm{CH}), 129.3(\mathrm{C}-\mathrm{N})$, $138.1(\mathrm{C}-\mathrm{Cl}), 133.2(\mathrm{CH}), 150.3(\mathrm{C}=\mathrm{N}), 152.4(\mathrm{C}=\mathrm{O}), 160.4$ (C-O), 176.5 (C=S); MS (m / z: \%): $394\left(\mathrm{M}^{+}, 22.7\right), 151$ (100); Anal. Calcd for $\mathrm{C}_{17} \mathrm{H}_{13} \mathrm{Cl}_{2} \mathrm{~N}_{3} \mathrm{O}_{2} \mathrm{~S}$ (394.28): $\mathrm{C}, 51.79 ; \mathrm{H}, 3.32 ; \mathrm{N}$, 10.66; Found: C, 51.74; H, 3.29; N, 10.73 .

\section{General Procedure for the Synthesis of 5-Imino-4-thioxoimidazolidin-2-one Derivatives $4 a, b$}

A solution of the cyanothioformanilide derivatives $(0.01 \mathrm{~mol})$ and 1,3-dichloro-5-isocyanatobenzene $(0.01 \mathrm{~mol})$ in dry ether $(30 \mathrm{~mL})$ was treated with three drops of triethylamine. The reaction mixture was stirred for $15 \mathrm{~min}$. The obtained product was filtered off, washed with ether, air-dried and recrystallized from chloroform / $n$-hexane to give imidazolidineiminothione derivatives $\mathbf{4 a}$ and $\mathbf{4 b}$.

\section{1-(2,6-DICHLOROPHENYL)-4-IMINO-3-PHENYL-5- THIOXOIMIDAZOLIDIN-2-ONE (4a)}

Yield $85 \%$; mp 194-196 ${ }^{\circ} \mathrm{C}$; IR (KBr): $v / \mathrm{cm}^{-1}=3420(\mathrm{NH})$, $1784(\mathrm{C}=\mathrm{O}), 1667(\mathrm{C}=\mathrm{N}), 1105(\mathrm{C}=\mathrm{S}) ;{ }^{1} \mathrm{H} \mathrm{NMR}\left(\mathrm{CDCl}_{3}, 400\right.$ $\mathrm{MHz}): \delta / \mathrm{ppm}=7.38-7.43(\mathrm{~m}, 1 \mathrm{H}, \mathrm{Ar}-\mathrm{H}), 7.59-7.46(\mathrm{~m}, 7 \mathrm{H}$, $\mathrm{Ar}-\mathrm{H}), 9.50(\mathrm{~s}, 1 \mathrm{H}, \mathrm{NH}) ;{ }^{13} \mathrm{C} \mathrm{NMR}\left(\mathrm{CDCl}_{3}, 100 \mathrm{MHz}\right): \delta / \mathrm{ppm}$ $=127.1(\mathrm{CH}) ; 127.9(\mathrm{C}), 128.9(\mathrm{CH}), 129.5(\mathrm{CH}), 129.8(\mathrm{CH})$, $131.7(\mathrm{CH}), 132.6(\mathrm{C}), 135.5(\mathrm{C}), 152.3(\mathrm{C}=\mathrm{O}$ and $\mathrm{C}=\mathrm{N}), 181.3$ (C=S), MS ( $\mathrm{m} / \mathrm{z}$ : \%): $350\left(\mathrm{M}^{+}, 3.4\right), 135$ (100); Anal. Calcd for $\mathrm{C}_{15} \mathrm{H}_{9} \mathrm{Cl}_{2} \mathrm{~N}_{3} \mathrm{OS}$ (350.22): C, 51.44; $\mathrm{H}, 2.59 ; \mathrm{N}, 12.00$; Found: $\mathrm{C}, 51.53 ; \mathrm{H}, 2.62 ; \mathrm{N}, 12.14$.

\section{1-(4-BROMOPHENYL)-3-(2,6-DICHLOROPHENYL)-5-} IMINO-4-THIOXOIMIDAZOLIDIN-2-ONE (4b)

Yield 83 \%; mp 207-209 ${ }^{\circ} \mathrm{C}$; IR: $v / \mathrm{cm}^{-1}$ : $3231(\mathrm{NH}), 1772$ $(\mathrm{C}=\mathrm{O}), 1644(\mathrm{C}=\mathrm{N}), 1125$ (C=S); ${ }^{1} \mathrm{H}$ NMR (d1-TFA $/ \mathrm{CDCl}_{3} ; 1 / 1$,
$400 \mathrm{MHz}): \delta / \mathrm{ppm}=7.42(\mathrm{~d}, 2 \mathrm{H}, J=8.8 \mathrm{~Hz}, \mathrm{Ar}-\mathrm{H}), 7.67(\mathrm{~m}$, $3 \mathrm{H}, \mathrm{Ar}-\mathrm{H}), 7.82(\mathrm{~d}, 2 \mathrm{H}, J=8.8 \mathrm{~Hz}, \mathrm{Ar}-\mathrm{H}), 9.52(\mathrm{br}, 1 \mathrm{H}, \mathrm{NH})$; ${ }^{13} \mathrm{C} \mathrm{NMR}$ (d1-TFA / $\mathrm{CDCl}_{3} ; 1 / 1,100 \mathrm{MHz}$ ): 123.5 (C-Br); 126.2 (C-N), $128.5(\mathrm{C}-\mathrm{H}), 129.8(\mathrm{C}-\mathrm{N}), 130.3(\mathrm{CH}), 134.1(\mathrm{CH})$, $135.0(\mathrm{C}-\mathrm{Cl}), 135.2(\mathrm{CH}), 149.7(\mathrm{C}=\mathrm{N}), 150.5(\mathrm{C}=\mathrm{O}), 175.1$ (C=S); MS ( $m$ / z: \%): $429\left(\mathrm{M}^{+}, 10.8\right), 124$ (100); Anal. Calcd for $\mathrm{C}_{15} \mathrm{H}_{8} \mathrm{BrCl}_{2} \mathrm{~N}_{3} \mathrm{OS}$ (429.12): C, 41.98; $\mathrm{H}, 1.88 ; \mathrm{N}, 9.79$; Found: $\mathrm{C}, 42.11 ; \mathrm{H}, 1.91 ; \mathrm{N}, 9.84$

\section{General Procedure for the Synthesis of 5-Imino-4-thioxoimidazolidin-2-one Derivatives 6a-c}

A solution of the cyanothioformanilide derivatives $(0.01 \mathrm{~mol})$ and (isocyanatomethyl)benzene $(0.01 \mathrm{~mol})$ in dry ether (30 $\mathrm{mL}$ ) was treated with three drops of triethylamine. The reaction mixture was stirred for $15 \mathrm{~min}$. The obtained product was filtered off, washed with ether, air-dried and recrystallized from chloroform / $n$-hexane to give imidazolidineiminothione derivatives $\mathbf{6 a}-\mathbf{c}$.

\section{1-BENZYL-5-IMINO-4-THIOXO-3-P-TOLYLIMIDAZOLIDIN-2- ONE (6a)}

Yield $80 \%$; mp 123-125 ${ }^{\circ} \mathrm{C}$; IR $(\mathrm{KBr}): \mathrm{v} / \mathrm{cm}^{-1}=3410(\mathrm{NH})$, $1780(\mathrm{C}=\mathrm{O}), 1668(\mathrm{C}=\mathrm{N}), 1082(\mathrm{C}=\mathrm{S})$; ${ }^{1} \mathrm{H} \mathrm{NMR}\left(\mathrm{CDCl}_{3}, 400\right.$ $\mathrm{MHz}): \delta / \mathrm{ppm}=2.40\left(\mathrm{~s}, 3 \mathrm{H}, \mathrm{CH}_{3}\right), 4.98\left(\mathrm{~s}, 2 \mathrm{H}, \mathrm{CH}_{2}\right), 7.20$ 7.38 (m, 7H, Ar-H), 7.48-7.54 (m, 2H, Ar-H), 9.36 (br, $1 \mathrm{H}$, $\mathrm{NH}) ;{ }^{13} \mathrm{C} \mathrm{NMR}\left(\mathrm{CDCl}_{3}, 100 \mathrm{MHz}\right): 21.3\left(\mathrm{CH}_{3}\right), 44.3\left(\mathrm{CH}_{2}\right)$, $126.7(\mathrm{CH}), 128.2(\mathrm{CH}), 128.7(\mathrm{CH}), 129.0(\mathrm{CH}), 130.0(\mathrm{CH})$, 130.1 (C), 135.3 (C), 139.7 (C), $153.7(\mathrm{C}=\mathrm{O}), 154.7(\mathrm{C}=\mathrm{N})$, 182.0 (C=S); MS (m / z: \%): $309\left(\mathrm{M}^{+}, 20.8\right), 91$ (100): Anal. Calcd for $\mathrm{C}_{17} \mathrm{H}_{15} \mathrm{~N}_{3} \mathrm{OS}$ (309.39): C, 66.00; H, 4.89; N, 13.58; Found: $\mathrm{C}, 66.12 ; \mathrm{H}, 4.92 ; \mathrm{N}, 13.67$.

\section{1-BENZYL-5-IMINO-3-(4-METHOXYPHENYL)-4- THIOXOIMIDAZOLIDIN-2-ONE (6b)}

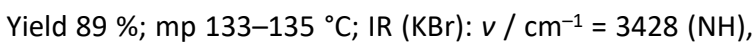
1775 (C=O), 1665 (C=N), 1254 (C-O), 1146 (C-O), 1086 $(\mathrm{C}=\mathrm{S}) ;{ }^{1} \mathrm{H}$ NMR $\left(\mathrm{CDCl}_{3}, 400 \mathrm{MHz}\right) \delta / \mathrm{ppm}=3.84(\mathrm{~s}, 3 \mathrm{H}$, $\left.\mathrm{OCH}_{3}\right), 4.98\left(\mathrm{~s}, 2 \mathrm{H}, \mathrm{CH}_{2}\right), 7.00(\mathrm{~d}, 2 \mathrm{H}, J=9.1 \mathrm{~Hz}, \mathrm{Ar}-\mathrm{H}), 7.27-$ $7.39(\mathrm{~m}, 5 \mathrm{H}, \mathrm{Ar}-\mathrm{H}), 7.49-7.54(\mathrm{~m}, 2 \mathrm{H}, \mathrm{Ar}-\mathrm{H}), 9.36(\mathrm{br}, 1 \mathrm{H}$, $\mathrm{NH}) ;{ }^{13} \mathrm{C} \mathrm{NMR}\left(\mathrm{CDCl}_{3}, 100 \mathrm{MHz}\right): 44.4\left(\mathrm{CH}_{2}\right) 55.5\left(\mathrm{CH}_{3}\right)$, $114.6(\mathrm{CH}), 125.3(\mathrm{C}), 128.2(\mathrm{CH}), 128.3(\mathrm{CH}), 128.7(\mathrm{CH})$, $129.0(\mathrm{CH}), 135.4(\mathrm{C}), 153.7(\mathrm{C}=\mathrm{O}), 154.9(\mathrm{C}=\mathrm{N}), 160.1(\mathrm{C}-$ O), 182.2 (C=S); $\mathrm{MS}$ ( $\mathrm{m} / \mathrm{z}$ : \%): $325\left(\mathrm{M}^{+}, 52.1\right), 51(100)$; Anal. Calcd for $\mathrm{C}_{17} \mathrm{H}_{15} \mathrm{~N}_{3} \mathrm{O}_{2} \mathrm{~S}$ (325.38): C, 62.75; H, 4.65; N, 12.91; Found: $\mathrm{C}, 62.84 ; \mathrm{H}, 4.73 ; \mathrm{N}, 12.84$

\section{1-BENZYL-3-(4-ETHOXYPHENYL)-5-IMINO-4- THIOXOIMIDAZOLIDIN-2-ONE (6c)}

Yield $83 \%$; mp 131-133 ${ }^{\circ} \mathrm{C}$; IR ( $\left.\mathrm{KBr}\right) 3435(\mathrm{NH}), 1775$ (C=O), $1667(\mathrm{C}=\mathrm{N}), 1250$ (C-O), 1138 (C-O), 1084 (C=S); ${ }^{1} \mathrm{H}$ NMR $\left(\mathrm{CDCl}_{3}, 400 \mathrm{MHz}\right): \delta / \mathrm{ppm}=1.43\left(\mathrm{t}, 3 \mathrm{H}, J=7.0 \mathrm{~Hz}, \mathrm{CH}_{2} \underline{\mathrm{CH}_{3}}\right)$, 4.06 (q, $2 \mathrm{H}, J=7.0 \mathrm{~Hz}, \underline{\mathrm{CH}_{2}} \mathrm{CH}_{3}$ ), 4.99 (s, 2H, $\left.\mathrm{CH}_{2}\right), 6.99$ (d, 
$2 \mathrm{H}, J=9.1 \mathrm{~Hz}, \mathrm{Ar}-\mathrm{H}), 7.24-7.39(\mathrm{~m}, 5 \mathrm{H}, \mathrm{Ar}-\mathrm{H}), 7.49-7.54$ (m, 2H, Ar-H), $9.35(\mathrm{br}, 1 \mathrm{H}, \mathrm{NH}) ;{ }^{13} \mathrm{C} \mathrm{NMR}\left(\mathrm{CDCl}_{3}, 100 \mathrm{MHz}\right)$ : $14.7\left(\mathrm{CH}_{3}\right), 44.4\left(\mathrm{CH}_{2}\right), 63.8\left(\mathrm{CH}_{2}\right), 115.1(\mathrm{CH}), 125.0(\mathrm{C})$, $128.1(\mathrm{CH}), 128.2(\mathrm{CH}), 128.7(\mathrm{CH}), 129.0(\mathrm{CH}), 135.4(\mathrm{C})$, $153.7(\mathrm{C}=\mathrm{O}), 154.9(\mathrm{C}=\mathrm{N}), 159.5$ (C-O), 182.2 (C=S); MS ( $\mathrm{m} /$ z: \%): 339 (M+, 29.4), 91 (100); Anal. Calcd for $\mathrm{C}_{18} \mathrm{H}_{17} \mathrm{~N}_{3} \mathrm{O}_{2} \mathrm{~S}$ (339.41): C, 63.70; H, 5.05; N, 12.38; Found: C, 63.62; H, $5.01 ; \mathrm{N}, 12.42$.

\section{Synthesis of Imidazolidin-2,5-diones 7a-c}

Each one of imidazolidineiminothione derivatives $6 a-c$ (0.01 mol) was dissolved in boiling ethanol $(20 \mathrm{~mL})$ and treated with dil. $\mathrm{HCl}(1: 1$ molar ratio). The obtained products were filtered off, washed with cold water, air-dried, and recrystallized from chloroform / $n$-hexane to give the corresponding diones $\mathbf{7 a - c}$.

\section{3-BENZYL-5-THIOXO-1-P-TOLYLIMIDAZOLIDINE-2,4- DIONE (7a)}

Yield $73 \%$; mp 105-106 ${ }^{\circ} \mathrm{C}$; IR (KBr): $v / \mathrm{cm}^{-1}=1740(\mathrm{C}=0)$,

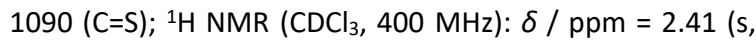
$\left.3 \mathrm{H}, \mathrm{CH}_{3}\right), 4.89\left(\mathrm{~s}, 2 \mathrm{H}, \mathrm{CH}_{2}\right), 7.21-7.25(\mathrm{~m}, 2 \mathrm{H}, \mathrm{Ar}-\mathrm{H}), 7.28-$ $7.33(\mathrm{~m}, 2 \mathrm{H}, \mathrm{Ar}-\mathrm{H})$, 7.34-7.40 (m, 3H, Ar-H), 7.46-7.52 (m, $2 \mathrm{H}, \mathrm{Ar}-\mathrm{H}) ;{ }^{13} \mathrm{C} \mathrm{NMR}\left(\mathrm{CDCl}_{3}, 100 \mathrm{MHz}\right): 21.3\left(\mathrm{CH}_{3}\right), 43.8$ $\left(\mathrm{CH}_{2}\right), 126.8(\mathrm{CH}), 128.6(\mathrm{CH}), 129.0(\mathrm{CH}), 129.2(\mathrm{CH}), 129.4$ (C), $130.1(\mathrm{CH}), 140.0$ (C), 134.5 (C), $153.6(\mathrm{C}=0), 154.1$ (C=O), 183.1 (C=S); MS ( $m$ / z: \%): $310\left(\mathrm{M}^{+}, 28.1\right), 91(100)$; Anal. Calcd for $\mathrm{C}_{17} \mathrm{H}_{14} \mathrm{~N}_{2} \mathrm{O}_{2} \mathrm{~S}$ (310.37): $\mathrm{C}, 65.79 ; \mathrm{H}, 4.55 ; \mathrm{N}$, 9.03; Found: $\mathrm{C}, 65.82 ; \mathrm{H}, 4.53 ; \mathrm{N}, 8.98$.

\section{3-BENZYL-1-(4-METHOXYPHENYL)-5- THIOXOIMIDAZOLIDINE-2,4-DIONE (7b)}

Yield $80 \%$; mp 104-105 ${ }^{\circ} \mathrm{C}$; IR $(\mathrm{KBr}): v / \mathrm{cm}^{-1}=1738(\mathrm{C}=0)$, 1252 (C-O), 1167 (C-O), 1094 (C=S); ${ }^{1} \mathrm{H}$ NMR $\left(\mathrm{CDCl}_{3}, 400\right.$ $\mathrm{MHz}): \delta / \mathrm{ppm}=3.84\left(\mathrm{~s}, 3 \mathrm{H}, \mathrm{OCH}_{3}\right) ; 4.88\left(\mathrm{~s}, 2 \mathrm{H}, \mathrm{CH}_{2}\right), 7.00$ (d, $2 \mathrm{H}, J=9.1 \mathrm{~Hz}, \mathrm{Ar}-\mathrm{H}), 7.26$ (d, $2 \mathrm{H}, J=9.1 \mathrm{~Hz}, \mathrm{Ar}-\mathrm{H}), 7.32$ $7.40(\mathrm{~m}, 3 \mathrm{H}, \mathrm{Ar}-\mathrm{H}), 7.46-7.51(\mathrm{~m}, 2 \mathrm{H}, \mathrm{Ar}-\mathrm{H}) ;{ }^{13} \mathrm{C}$ NMR $\left(\mathrm{CDCl}_{3}, 100 \mathrm{MHz}\right): 43.8\left(\mathrm{CH}_{2}\right) ; 55.5\left(\mathrm{CH}_{3}\right), 114.7(\mathrm{CH}), 124.5$ (C), $128.3(\mathrm{CH}), 128.6(\mathrm{CH}), 129.0(\mathrm{CH}), 129.2(\mathrm{CH}), 134.5$ (C), $153.7(\mathrm{C}=\mathrm{O}), 154.2(\mathrm{C}=\mathrm{O}), 160.2(\mathrm{C}-\mathrm{O}), 183.2(\mathrm{C}=\mathrm{S}), \mathrm{MS}$ (m / z: \%): $326\left(\mathrm{M}^{+}, 45\right), 91(100) ;$ Anal. Calcd for $\mathrm{C}_{17} \mathrm{H}_{1} 4 \mathrm{~N}_{2} \mathrm{O}_{3} \mathrm{~S}$ (326.37): C, 62.56; $\mathrm{H}, 4.32 ; \mathrm{N}, 8.58$; Found: $\mathrm{C}$, $62.62 ; \mathrm{H}, 4.30 ; \mathrm{N}, 8.64$.

\section{3-BENZYL-1-(4-ETHOXYPHENYL)-5-}

\section{THIOXOIMIDAZOLIDINE-2,4-DIONEONE (7c)}

Yield $88 \%$; $\mathrm{mp} 120-121^{\circ} \mathrm{C}$; IR $(\mathrm{KBr}): \mathrm{v} / \mathrm{cm}^{-1}=1744(\mathrm{C}=0)$, 1250 (C-O), 1169 (C-O), 1088 (C=S); ${ }^{1} \mathrm{H}$ NMR (CDCl, 400 $\mathrm{MHz}): \delta / \mathrm{ppm}=1.43(\mathrm{t}, 3 \mathrm{H}, J=7.0 \mathrm{~Hz}),, 4.06(\mathrm{q}, J=7.0 \mathrm{~Hz}$, $2 \mathrm{H}), 4.88(\mathrm{~s}, 2 \mathrm{H}), 6.98(\mathrm{~d}, J=9.0 \mathrm{~Hz}, 2 \mathrm{H}), 7.25(\mathrm{~d}, J=9.0 \mathrm{~Hz}$, 2H), 7.31-7.40 (m, 3H, Ar-H), 7.45-7.51 (m, 2H, Ar-H); ${ }^{13} \mathrm{C}$ NMR $\left(\mathrm{CDCl}_{3}, 100 \mathrm{MHz}\right): 14.7\left(\mathrm{CH}_{3}\right), 43.8\left(\mathrm{CH}_{2}\right), 63.8\left(\mathrm{CH}_{2}\right)$, $115.2(\mathrm{CH}), 124.3(\mathrm{C}), 128.2(\mathrm{CH}), 128.6(\mathrm{CH}), 129.2(\mathrm{CH})$
$130.0(\mathrm{CH}), 134.5(\mathrm{C}), 153.7(\mathrm{C}=0), 154.3(\mathrm{C}=0), 159.6$ (CO), 183.2 (C=S); $\mathrm{MS}$ ( $\mathrm{m} / \mathrm{z}$ : \%): $340\left(\mathrm{M}^{+}, 17.8\right), 91$ (100); Anal. Calcd for $\mathrm{C}_{18} \mathrm{H}_{16} \mathrm{~N}_{2} \mathrm{O}_{3} \mathrm{~S}$ (340.40): $\mathrm{C}, 63.51 ; \mathrm{H}, 4.74 ; \mathrm{N}, 8.23$; Found: $\mathrm{C}, 63.47 ; \mathrm{H}, 4.77 ; \mathrm{N}, 8.34$

\section{Antimicrobial Screening}

MICs were assessed by serial broth dilution method. ${ }^{[12]}$ The freshly prepard inocula were incubated for $24 \mathrm{~h}$ at $37^{\circ} \mathrm{C}$ in NB for bacteria and $48 \mathrm{~h}$ at $30^{\circ} \mathrm{C}$ in Sabouraud dextrose broth for fungi. The chemical compounds were first dissolved in DMSO and then different concentrations of them were prepared by diluting the samples in NB and Sabouraud dextrose broth for bacteria and fungi, respectively to reach the final concentration of 1 to $100 \mu \mathrm{gL}^{-1}$. Each one $\mathrm{mL}$ from the above concentrations received $0.1 \mathrm{~mL}$ of the inoculum to reach $10^{6} \mathrm{CFU} \mathrm{mL}-1$ for bacteria and fungi. Triplicate tests were performed and the average was taken as the final reading comparing to negative and positive controls.

\section{RESULTS AND DISCUSSION}

\section{Chemistry}

Cyanothioformamide derivative 1 was prepared by reaction 3,5-dichlorophenylisothiocyanate with potassium cyanide according to literature procedure. ${ }^{[5]}$ The tautomeric nature of the $\mathrm{N}$-arylcyanothioformamide reflects their nucleophilic character in which they may react via the nitrogen or sulfur atom leading to different ring closing reactions. Reaction of $\mathrm{N}$-arylcyanothioformamide derivative 1 with various isocyanate derivatives in ether, followed by addition of a few drops of triethylamine as catalyst afforded imidazolidineiminothione derivatives $\mathbf{2 a}-\mathbf{h}$ (Scheme 1). Their IR, NMR $\left({ }^{1} \mathrm{H}\right.$ and $\left.{ }^{13} \mathrm{C}\right)$ and mass spectra indicated that 5-imino-4-thioxo-2-imidazolidinone derivatives $\mathbf{2} \mathbf{a}-\mathbf{h}$ were furnished as the sole products, indicating that the ring closing reaction proceeds via a single path which involves attack via the nitrogen atom.

It has been reported that moving side chain around the the main scaffold to the other position can result in retention of biological activities and providing new opportunities for the design of bioactive compounds. ${ }^{[11]}$ These observations gave us an additional motivation to combination of the 3,5-dichlorophenyl moiety with imidazole scaffold at $\mathrm{N}-(1)$ of the imidazole. Therefore, treating an ethereal solution of $\mathrm{N}$-arylcyanothioformamides $\mathbf{3} \mathbf{a}, \mathbf{b}$ with 3,5-dichlorophenylisocyanate, followed by addition of a few drops of triethylamine afforded imidazolidineiminothione derivatives $\mathbf{4 a , b}$.

Moreover, our investigation was extended to include the behavior of benzylisothiocyanate toward different $\mathrm{N}$ arylcyanothioformamides. Thus, when $\mathrm{N}$-arylcyanothiofor- 
mamides $\mathbf{5 a - c}$ were let to react with benzylisothiocyanate in ether in precence of a few drops of triethylamine, imidazolidineiminothione derivatives $\mathbf{6 a - c}$ were afford in good yeilds. The structure of compounds $6 \mathbf{a}-\mathbf{c}$ was inferred from their correct elemental analyses and spectral data. To obtain another type of imidazole, hydrolysis of $\mathbf{6 a - c}$ with dilute $\mathrm{HCl}$ was carredout in boiling ethanol and the corresponding dione derivatives $7 \mathrm{a}-\mathbf{c}$ were abtianed (Scheme 3 ).

\section{Biological Activity}

The aim of the present investigation is to synthesize series of imidazolidineiminothiones was bearing various substituent at $\mathrm{N}-(1)$ and others at $\mathrm{N}-(3)$. The antibacterial and antifungal activities of these derivatives were measured. The effect of each substituent at $\mathrm{N}$-(1) and at $\mathrm{N}$-(3) on these activities was studied and make a comparative study between them to deduce a structure activity relationship was made. A series of imidazolidineiminothione $\mathbf{2} \mathbf{a}-\mathbf{h}$ containing 3,5-dichlorophenyl moiety at $\mathrm{N}$-(1) and various aryl at N-(3) was synthesized. The effect of each substituent on $\mathrm{N}$-(3) of the imidazolidine ring on the activities of the imidazoslidines $\mathbf{2} \mathbf{a}-\mathbf{h}$ was studied. Thus, changing the

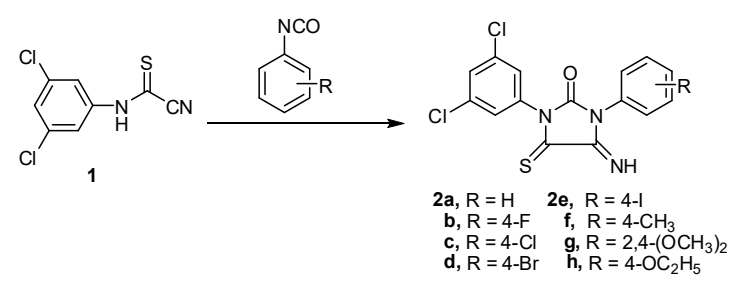

Scheme 1. Synthesis of imidazolidineiminothione derivatives $2 \mathrm{a}-\mathrm{h}$.
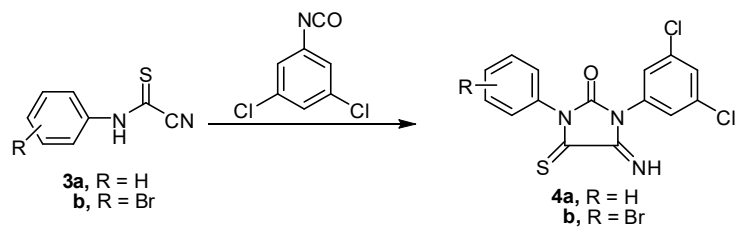

Scheme 2. Synthesis of imidazolidineiminothione derivatives $4 a, b$.

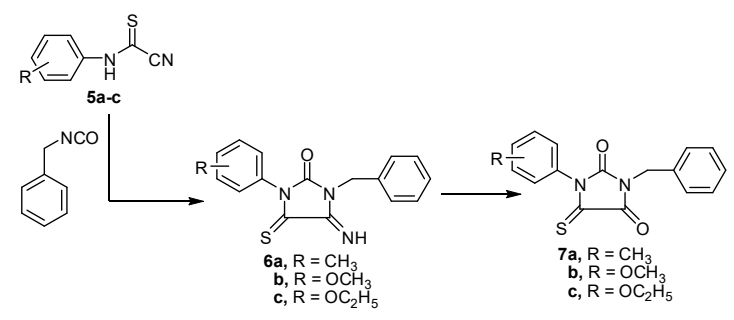

Scheme 3. Synthesis of imidazolidineiminothione derivatives 6a-c and imidazolidin-2,5-dione derivatives 7a-c substituent on $\mathrm{N}$-(3) from phenyl to 4-fluorophenyl, 4chlorophenyl, 4-bromophenyl, 4-iodophenyl, 4-tolyl, 2,4dimethoxyphenyl and 4-ethoxyphenyl (2a, 2b, 2c, 2d, 2e, $\mathbf{2 f}, \mathbf{2} \mathbf{g}$ and $\mathbf{2 h}$ ) was carried out to show the difference between $\mathrm{F}, \mathrm{Cl}, \mathrm{Br}, \mathrm{I}, \mathrm{CH}_{3}, \mathrm{OCH}_{3}$ and $\mathrm{OC}_{2} \mathrm{H}_{5}$ on the effect of the biological activities. Inverse the substituent at $\mathrm{N}$-(1) and $\mathrm{N}$-(3) was carried out where 3,5-dichlorophenyl moiety was attached to $\mathrm{N}-(3)$ instead if $\mathrm{N}-(1)$, and phenyl (4a) and 4bromophenyl (4b) was attached to $\mathrm{N}-(1)$ instead of $\mathrm{N}-(3)$, in order to study the difference of biological activity between $\mathbf{2 a}$ and $\mathbf{4 a}, \mathbf{2} \mathbf{d}$ and $\mathbf{4} \mathbf{b}$. Another imidazolidineiminothiones $6 \mathrm{a}-\mathrm{c}$ which contain benzyl moiety at $\mathrm{N}-(1)$ and various moieties at $\mathrm{N}-(3)$ were synthesized. Changing the substituent on $\mathrm{N}$-(3) from $p$-tolyl, $p$-anizyl and $p$-ethoxyphenyl $(\mathbf{6} a, \mathbf{6 b}$ and $\mathbf{6 c}$ ) was carried out. Moreover, the imine functional group in $6 a-c$ was changed to carbonyl functional group in $7 \mathrm{a}-\mathrm{c}$ while the substituent at $\mathrm{N}-(1)$ and $\mathrm{N}-(3)$ is not changed.

\section{Antibacterial and Antifungal Activities}

The newly synthesized target compounds were evaluated in vitro for their expected antimicrobial activity. Nine test organisms representing three different microbial groups were used: three Gram-positive bacteria, Bacillus subtilis ATCC 6633, B. cereus ATCC6629 and Staphylococcus aureus ATCC 29213; four Gram-negative bacteria, Klebseilla peneumoniae ATCC 13883, Escherichia coli ATCC 25922, Proteous vulgaris and salmonella typhimurium ATCC14028; fungi, Saccharomyces cerevisiae (local isolate) and Candida albicans ATCC10231. Ciprofloxacin and ketoconazole were used as standard references for antibacterial and antifungal activity, respectively. The minimal inhibitory concentrations (MICs) for the synthesized compounds were assessed by serial broth dilution method ${ }^{[12]}$ and the MIC results $\left(\mu \mathrm{g} \mathrm{mL}^{-1}\right)$ were presented in Table 1.

Generaly, the mean values of the obtained MIC suggest that most of the imidazole derivatives evaluated possess significant antibacterial activity against $\mathrm{G}+\mathrm{ve}$ bacteria and no results against $G$-ve bactria. Also, most of the imidazole derivatives evaluated possess significant antifungal. A moderate difference in the antimicrobial activity is noted between the tested compounds. This suggested that, the main effect may be related to the presence of the imidazolidinethione moiety. All of the imidazole derivatives possess significant antifungal aginst S. cerevisiae MIC (1-10 $\left.\mu \mathrm{g} \mathrm{mL}^{-1}\right)$. As comparision with ketoconazole, the imidazole derivatives showed activity ranged from $50 \%$ less activity to fourfold activity.

\section{CONCLUSION}

The synthesis, characterization and evaluation of antibacterial and antifungal activities of new series of 1,3- 
Table 1. Minimum inhibitory concentration $\left(\mu \mathrm{g} \mathrm{mL}^{-1}\right)$ of the synthesized compounds against the pathological organisms.

\begin{tabular}{|c|c|c|c|c|c|c|c|c|c|}
\hline \multirow{2}{*}{$\begin{array}{c}\text { Compd. } \\
\text { no }\end{array}$} & \multicolumn{3}{|c|}{ Gram +ve } & \multicolumn{4}{|c|}{ Gram-ve } & \multicolumn{2}{|c|}{ Fungi } \\
\hline & B. subtilis & B. cereus & S. aureus & K. pneumonia & E. coli & P. vulgaris & Salmonella & S. cerevisiae & C. albicans \\
\hline $2 a$ & 30 & $>500$ & 50 & $>500$ & $>500$ & $>500$ & $>500$ & 4 & 20 \\
\hline $2 b$ & $>500$ & $>500$ & 50 & $>500$ & $>500$ & $>500$ & $>500$ & 10 & 20 \\
\hline $2 c$ & 10 & $>500$ & 10 & $>500$ & $>500$ & $>500$ & $>500$ & 10 & 20 \\
\hline $2 d$ & 20 & 30 & 20 & $>500$ & $>500$ & $>500$ & $>500$ & 10 & 1 \\
\hline $2 \mathrm{e}$ & 20 & $>500$ & 10 & $>500$ & $>500$ & $>500$ & $>500$ & 4 & 25 \\
\hline $2 f$ & 30 & 20 & 40 & $>500$ & $>500$ & $>500$ & $>500$ & 10 & 20 \\
\hline $2 \mathrm{~g}$ & 20 & $>500$ & 40 & $>500$ & $>500$ & $>500$ & $>500$ & - & 30 \\
\hline $2 \mathrm{~h}$ & $>500$ & 20 & $>500$ & $>500$ & $>500$ & $>500$ & $>500$ & 10 & 20 \\
\hline $4 a$ & 20 & 30 & 40 & $>500$ & $>500$ & $>500$ & $>500$ & 4 & 30 \\
\hline $4 b$ & 25 & 30 & 40 & $>500$ & $>500$ & $>500$ & $>500$ & 4 & 30 \\
\hline $6 a$ & 30 & $>500$ & 40 & $>500$ & $>500$ & $>500$ & $>500$ & 10 & 30 \\
\hline $6 b$ & 20 & $>500$ & 50 & $>500$ & $>500$ & $>500$ & $>500$ & 1 & 20 \\
\hline $6 c$ & 20 & 30 & 40 & $>500$ & $>500$ & $>500$ & $>500$ & 10 & 20 \\
\hline $7 a$ & 2 & 30 & 50 & $>500$ & $>500$ & $>500$ & $>500$ & 10 & 30 \\
\hline $7 b$ & 20 & 30 & 50 & $>500$ & $>500$ & $>500$ & $>500$ & 1 & 30 \\
\hline $7 c$ & 20 & 20 & 50 & $>500$ & $>500$ & $>500$ & $>500$ & 4 & 30 \\
\hline CFL & 10 & 10 & 11 & - & - & - & - & - & - \\
\hline KET & - & - & - & - & - & - & - & 5 & 10 \\
\hline
\end{tabular}

disubstituted-4-thioxoimidazolidin-2-one derivatives with variable substituents at $\mathrm{N}-(1)$ and $\mathrm{N}-(3)$ have been described. Some compounds displayed antibacterial and antifungal activities. Generaly, the mean values of the obtained MIC suggest that some of the imidazole derivatives evaluated possess significant antibacterial activity against $\mathrm{G}+$ ve bacteria and no activity against $\mathrm{G}$-ve bacteria. Also, some of the imidazole derivatives possess significant antifungal effect. A moderate difference in the antimicrobial activity is noted between the tested compounds. This suggested that, the main effect may be related to the presence of the imidazolidinethione moiety.

\section{REFERENCES}

[1] B. B. Trunz, R. Jedrysiak, D. Tweats, R. Brun, M. Kaiser, J. Snski, E. Torreele, Eur. J. Med. Chem. 2011, 46, 1524.

[2] C. D. Freeman, N. E. Klutman, K. C. Lamp, Drugs 1997, 54, 679.

[3] Y. A. Ammar, S. Y. Abbas, M. A. M. Sh. El-Sharief, M. A. Salem, A. Ragab, Eur. J. Chem. 2017, 8, 76.

[4] Y. A. Ammar, M. A. M. Sh. El-Sharief, M. M. Ghorab,
Y. A. Mohamed, A. Ragab, S. Y. Abbas, Cur. Org. Syn. 2016, 13, 466.

[5] M. A. M. Sh. El-Sharief, S. Y. Abbas, M. A. Zahran, Y. A. Mohamed, A. Ragab, Y. A. Ammar, Z. Naturforsch. B 2016, 71, 875.

[6] Z. Moussa, M. A. M. S. El-Sharief, S. Y. Abbas, Eur. J. Med. Chem. 2016, 122, 419.

[7] A. I. A. Khodair, E. S. H. El-Ashry, N. A. L. Al-Masoudi, Monatsh. Chem. 2004, 135, 1061.

[8] S. A. Hessein, M. A. M. El-Sharief, S. Y. Abbas, H. K. Thabet, Y. A. Ammar, Croat. Chem. Acta 2016, 89 (1), 91.

[9] S. A. Fouad, S. A. Hessein, S. Y. Abbas, A. M. Farrag, Y. A. Ammar, Croat. Chem. Acta 2018, 91, 99.

[10] S. Y. Abbas, W. M. Basyouni, K. A. M. El-Bayouk, Applied Organometallic Chemistry 2018, 32, e4032.

[11] M. F. El Shehry, M. M. Ghorab, S. Y. Abbas, E. A. Fayed, S. A. Shedid, Y. A. Ammar, Eur. J. Med. Chem. 2018, 143, 1463.

[12] P. R. Murray, E. J. Baron, M. A. Pfaller, F. C. Tenover, R. H. Yolki, Manual of clinical microbiology, $6^{\text {th }}$ Edn. American Society for Microbiology Washington, DC, 1995, p. 282. 